Revista Científica General José María Córdova

(Revista Colombiana de Estudios Militares y Estratégicos)

Bogotá D.C., Colombia

ISSN 1900-6586 (impreso), 2500-7645 (en línea)

Web oficial: https://www.revistacientificaesmic.com

\title{
La interrelación de derechos humanos y medio ambiente desde el concepto de la seguridad humana
}

\author{
Mauricio Antonio Torres Guarnizo \\ https://orcid.org/0000-0003-0487-7283 \\ mauricio.torres@esdegue.edu.co \\ Escuela Superior de Guerra "General Rafael Reyes Prieto”, Bogotá D.C., Colombia \\ Elmers Freddy Velandia Pardo \\ https://orcid.org/0000-0003-1217-9178 \\ elmers.velandia@esdegue.edu.co \\ Escuela Superior de Guerra "General Rafael Reyes Prieto", Bogotá D.C., Colombia
}

Citación APA: Torres Guarnizo, M. A., \& Velandia Pardo, E. F. (2022). La interrelación de derechos humanos y medio ambiente desde el concepto de la seguridad humana. Revista Científica General José María Córdova, 20(37), 111-128.

https://dx.doi.org/10.21830/19006586.803

Publicado en línea: $1 .^{\circ}$ de enero de 2022

Los artículos publicados por la Revista Cientifica General José María Córdova son de acceso abierto bajo una licencia Creative Commons: Atribución - No Comercial - Sin Derivados.

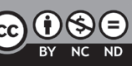

Para enviar un artículo:

https://www.revistacientificaesmic.com/index.php/esmic/about/submissions
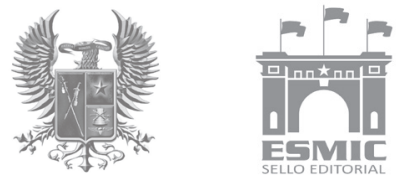

Miles Doctus 


\section{La interrelación de derechos humanos y medio ambiente desde el concepto de la seguridad humana}

\section{The interrelationship of human rights and the environment from the human security perspective}

\section{Mauricio Antonio Torres Guarnizo y Elmers Freddy Velandia Pardo}

Escuela Superior de Guerra "General Rafael Reyes Prieto", Bogotá D.C., Colombia

RESUMEN. Este artículo hace un análisis acerca de los derechos humanos y el medio ambiente en relación con la seguridad humana, a partir de la evolución del concepto de seguridad hasta alcanzar la perspectiva multidimensional adoptada en el plano internacional. A partir de las dimensiones de la seguridad humana, especialmente la dimensión de la seguridad ambiental, se estudia su interrelación con los derechos humanos. Para ello se analizan los principios marco sobre derechos humanos y medio ambiente planteados por Naciones Unidas en 2018, que establecen las principales obligaciones de los Estados en cuanto a los derechos humanos, relacionadas con el disfrute de un medio ambiente seguro, limpio, saludable y sostenible. Se concluye que estos principios marco son claves para armonizar y destacar la interrelación de los derechos humanos y el medio ambiente.

Palabras Clave: derechos humanos, desarrollo sostenible, medio ambiente, seguridad ambiental, seguridad humana

AвSTRACT. This article analyzes human rights and the environment as they relate to human security, starting from the evolution of the concept of security to the multidimensional perspective adopted internationally. Based on the dimensions of human security, especially the dimension of environmental security, it studies its interrelation with human rights. To this end, it analyzes the framework principles on human rights and the environment set forth by the United Nations in 2018, which establish the states' main obligations in terms of human rights, related to the enjoyment of a safe, clean, healthy, and sustainable environment. It concludes that these framework principles are key to harmonizing and highlighting the interrelationship of human rights and the environment.

KeYwORDS: environment; environmental security; human rights; human security; sustainable development

Sección: Justicia y Derechos Humanos • Artículo de investigación científica y tecnológica

Recibido: 29 de abril de 2021 • Aceptado: 22 de septiembre de 2021

ConTACTO: Mauricio Antonio Torres Guarnizo $\bigotimes$ mauricio.torres@esdegue.edu.co 


\section{Introducción}

A finales del siglo XX, el Programa de las Naciones Unidas para el Desarrollo (PNUD) (1994) planteó el concepto de seguridad humana, que concibe al individuo como eje central de la seguridad por encima del Estado mismo, como tradicionalmente se entendía desde finales de la Segunda Guerra Mundial bajo la perspectiva de la denominada seguridad nacional. La seguridad humana "es un concepto integrador" (PNUD, 1994, p. 27), de carácter universal, centrado en la vida y la dignidad de los individuos, y de carácter preventivo, cuyas dimensiones o componentes son interdependientes entre sí (Morrillas, 2006).

El objeto esencial de la seguridad humana consiste en la protección de los individuos frente a amenazas graves y generalizadas, que lesionen o tengan la capacidad de lesionar, a gran escala y/o de manera reiterada, la vida o el conjunto de funciones vitales de las personas (Alkire, 2003), en los ámbitos económico, alimentario, de la salud, ambiental, personal, comunitario y político (PNUD, 1994). Estas amenazas se pueden relacionar con la pobreza, el hambre, las enfermedades contagiosas, el deterioro ambiental, la violencia en sus diferentes expresiones y la represión política, entre otros factores (Mack, 2005).

El desarrollo, seguido por los derechos humanos y luego por la paz y la seguridad, son los pilares fundamentales de la Organización de Naciones Unidas (ONU). Estos pilares son interdependientes, de modo que deben fortalecerse en similar medida porque se refuerzan entre sí (ONU, 2012). Teniendo en cuenta esto, es evidente que existen diferentes puntos de conexión entre la seguridad humana, los derechos humanos y el desarrollo sostenible, de los cuales se destaca la protección al medio ambiente. Por una parte, los procesos de desarrollo no deben centrarse exclusivamente en lo económico, sino que deben considerar los aspectos sociales y ecológicos. Por otra parte, el derecho a un medio ambiente sano hace parte de los denominados derechos humanos de tercera generación. Finalmente, en relación con la paz y la seguridad, la seguridad humana tiene un componente denominado seguridad ambiental.

A pesar de ello, esta relación no se había materializado de manera evidente ni significativa en políticas, principios o normas que llevaran a constituir un verdadero sistema de protección y garantía al medio ambiente. Solo hasta la construcción de los Objetivos de Desarrollo Sostenible (ODS) (PNUD, 2015), se intentó incorporar y vincular esos tres pilares a la política internacional y, en consecuencia, a su inclusión en las agendas de cada Estado. Sin embargo, fue apenas hasta hace un par de años cuando el relator especial de las Naciones Unidas sobre los Derechos Humanos y el Medio Ambiente sistematizó una serie de obligaciones básicas de los Estados en lo relativo a los derechos humanos y su relación con el disfrute de un medio ambiente sin riesgos, limpio, saludable y sostenible, a través de unos Principios Marco sobre los Derechos Humanos y el Medio Ambiente -Oficina del Alto Comisionado para los Derechos Humanos (ACNUDH) (2018)_. Al parecer, estos principios no han trascendido de manera significativa en la comunidad internacional. 
Desde la Declaración Universal de Derechos Humanos por la ONU (1948), los derechos humanos han tenido un desarrollo constante, pasando por el derecho al medio ambiente en torno al desarrollo sostenible planteado por la Comisión Brundtland (1987) en el informe denominado Nuestro futuro común y la evolución del concepto de seguridad hasta llegar a la seguridad humana (PNUD, 1994). Así las cosas, no deja de sorprender que solo hasta hace unos pocos años se hizo el esfuerzo concreto de interrelacionar todos sus elementos y dimensiones con la formulación de los ODS en 2015 y de dichos principios marco en 2018. Teniendo esto en cuenta, se plantea el siguiente interrogante: ¡en qué medida se encuentran materialmente relacionados los principios marco con las dimensiones de la seguridad humana? Para resolver esta pregunta, primero se procede a analizar el vínculo existente entre derechos humanos y medio ambiente desde la óptica nacional e internacional; luego se estudia la relación concreta entre los derechos ambientales y las dimensiones de la seguridad humana, y finalmente se establece la relación material entre los principios marco y la seguridad humana.

Este artículo es resultado de una investigación aplicada desde un enfoque cualitativo, que pretende analizar, en una perspectiva analítica y crítica, la materialización de las dimensiones de la seguridad humana en torno a la normativa de derechos humanos frente al medio ambiente, a través de la revisión documental de los instrumentos internacionales, la normativa, la jurisprudencia y la doctrina en la materia.

\section{Derechos humanos y medio ambiente}

La formulación de un Estado social de derecho implica no solo la sujeción de la actividad estatal a las normas jurídicas, con base en la Constitución Política, sino que implica que el esfuerzo estatal debe dirigirse a garantizar condiciones de vida dignas para las personas. Por lo tanto, el individuo no debe limitarse a exigir al Estado que no haya interferencia en sus libertades, sino que además debe exigirle que "se ponga en movimiento para contrarrestar las desigualdades sociales existentes y para ofrecerle a todos las oportunidades necesarias para desarrollar sus aptitudes y para superar los apremios materiales" (Corte Constitucional, 1998, Sentencia SU-747).

En este sentido, la incidencia de este tipo de Estado en la organización sociopolítica se puede entender desde dos perspectivas: una cuantitativa, relacionada con el Estado de bienestar, y otra cualitativa, relativa al Estado constitucional democrático. Desde la perspectiva cuantitativa, el Estado debe garantizar estándares mínimos de salario, alimentación, salud y educación para todos los ciudadanos, como verdaderos derechos y no como un regalo. Por su parte, desde la perspectiva cualitativa, el Estado constitucional democrático "ha sido la respuesta jurídico-política derivada de la actividad intervencionista del Estado [...] fundada en nuevos valores-derechos consagrados por la segunda y tercera generación de derechos humanos" (Corte Constitucional, 1992, Sentencia T-406). Esto se traduce en la incorporación de principios y de derechos que inciden en el funcionamiento 
de la organización política, el establecimiento de mecanismos de democracia participativa y el ejercicio del control político y jurídico en el ejercicio del poder público.

De acuerdo con Landa (2017), el Estado de bienestar tuvo que asumir, por una parte, todas las cargas sociales que no fueron resueltas por el libre mercado y, por otra parte, asignar ciertas obligaciones a los grandes propietarios con la consagración de los derechos sociales y económicos para los trabajadores, lo que significó un impulso constante al crecimiento económico como base para la superación de la pobreza, pero fundamentado, desafortunadamente, en la destrucción de la naturaleza. Así las cosas, el desarrollo se alcanzó, durante mucho tiempo, ligado casi exclusivamente al aspecto netamente económico, al asumirse que con el crecimiento económico se alcanzaba el desarrollo per se. Esto produjo una verdadera tensión entre crecimiento económico y medio ambiente (Delgado, 1993).

Es indiscutible el carácter paradójico de esta situación. El desarrollo ha consistido siempre en dominar la naturaleza "para crear un ambiente artificial propiamente humano y por ello más cómodo para vivir" (Canosa, 2006, p. 153); pero alcanzar este punto solo fue posible con la disposición de todo aquello que es producido con la transformación, explotación y, en algunos casos, destrucción de los recursos naturales (Krämer, 1999). Hoy en día, es innegable que el derecho al medio ambiente se encuentra intrínsecamente relacionado con el desarrollo social y económico, de manera que no ponga en peligro el bien jurídico protegido del medio ambiente (Muñoz, 2017, p. 89). En este escenario, teniendo en cuenta que el enfoque basado en los derechos humanos (EBDH) promulgado por la ONU proporciona un marco conceptual fundamentado en las normas internacionales y orientado a la promoción y protección de los derechos humanos, el medio ambiente se convirtió precisamente en un bien jurídico protegido por la comunidad internacional.

Así, desde el EBDH, que "tiene como propósito analizar las obligaciones y desigualdades que se encuentran en el centro de los problemas de desarrollo, y corregir las prácticas discriminatorias y el injusto reparto del poder que obstaculizan el progreso en materia de desarrollo" (ACNUDH \& Cepal, 2016, p. 19), el medio ambiente se empezó a entender como un elemento esencial, transversal desde la perspectiva de los derechos humanos para el desarrollo y la seguridad.

Comprender al medio ambiente como bien jurídico protegido en los ordenamientos jurídicos no ha sido nuevo. Acero (2009) manifiesta que se ha llegado a verificar que desde el Imperio Romano ya existía una gran visión de protección ambiental. No obstante, los doctrinantes han coincidido en señalar que la Declaración de las Naciones Unidas sobre el Medio Humano (ONU, 1972) constituyó la fuente jurídica primaria que dio reconocimiento al medio ambiente como derecho (De Castro, 2003), al señalar en su Principio 1 que "el hombre tiene derecho fundamental a la libertad, la igualdad y el disfrute de condiciones de vida adecuadas en un medio ambiente de calidad tal que le permita llevar una vida digna y gozar de bienestar" (ONU, 1972). No obstante, también le asignó al 
individuo, y en consecuencia al Estado, "la solemne obligación de proteger y mejorar el medio ambiente para las generaciones presentes y futuras", entendiendo goce del medio ambiente como un derecho y su protección como un deber.

Sin embargo, no se puede desconocer que, en instrumentos internacionales previos, ya se había producido un reconocimiento indirecto al derecho al medio ambiente. Por ejemplo, la Declaración Universal de Derechos Humanos (1948), en su artículo 25 señala que "toda persona tiene el derecho a un nivel de vida adecuado que le asegure, así como a su familia, la salud y el bienestar". Así mismo, el Pacto Internacional de Derechos Económicos, Sociales y Culturales (ONU, 1966), en su artículo 12, se refiere al mejoramiento del medio ambiente como requisito para asegurar el goce efectivo del derecho a la salud tanto desde el aspecto físico como mental de las personas.

Por su parte, la doctrina ha considerado el derecho al medio ambiente como parte de la tercera generación de derechos (Vasak, 1984), fundamentados en la solidaridad frente a la búsqueda de mecanismos necesarios para mejorar las condiciones de vida de los individuos, el fomento del progreso social de la comunidad y el estímulo al desarrollo sostenible de los Estados. Es por ello que a estos derechos se les suele llamar derechos de los pueblos, derechos del medio ambiente o derecho al desarrollo sostenible (Pérez, 2007, p. 8). Esta tercera generación está compuesta por los derechos al medio ambiente, al desarrollo, a la paz, a la autodeterminación de los pueblos y al patrimonio común de la humanidad (Rodríguez, 2002).

En este marco, puede entenderse el medio ambiente como el conjunto de circunstancias que rodean e involucran a un entorno y que, de una u otra manera, influyen directamente en él. Según Pérez (2007), esto se refiere principalmente a dos tipos de circunstancias: las primeras, de carácter natural o físico de un determinado lugar, referidas a los recursos naturales que son necesarios para el desarrollo de la vida y que, de forma directa, repercuten e impactan en el desarrollo humano; las segundas circunstancias, generadas por la acción del hombre en su entorno, incluyen todas las circunstancias culturales, económicas y sociales en que vive un individuo en un determinado espacio, que influyen directamente en su desarrollo integral como persona en condiciones dignas.

Por ello, la doctrina ha señalado que el derecho a un ambiente sano es de índole tanto individual como colectivo, por lo cual se considera un derecho de incidencia colectiva (Rodríguez, 2017). En Colombia, la Corte Constitucional (1992), en Sentencia $\mathrm{T}-415$, ha manifestado que el derecho al medio ambiente se entiende como "un conjunto de condiciones básicas que rodean al hombre, que circundan su vida como miembro de la comunidad y que le permiten su supervivencia biológica e individual, además de su desempeño normal y desarrollo integral en el medio social". Es importante tener en cuenta que el Estado colombiano fue pionero en reconocer el medio ambiente como un bien jurídico protegido, tal como se evidencia en el artículo 2 del Decreto 2811 de 1974: "el ambiente es patrimonio común de la humanidad y necesario para la supervivencia y el desarrollo económico y social de los pueblos”. 
Así, en la Constitución Política de Colombia (1991), el medio ambiente se establece como un principio fundante del orden constitucional, al consagrar la obligación del Estado y de las personas a proteger las riquezas culturales y naturales de la Nación (Constitución Política, 1991, art. 8). Así mismo, se entiende como un derecho, al señalar que "todas las personas tienen derecho a gozar de un ambiente sano" (Constitución Política, 1991, art. 79). Por otra parte, le atribuye al Estado una serie de deberes y obligaciones: "proteger la diversidad e integridad del ambiente, conservar las áreas de especial importancia ecológica y fomentar la educación para el logro de estos fines" (Constitución Política, 1991, art. 79); "planificar el manejo y aprovechamiento de los recursos naturales, para garantizar su desarrollo sostenible, su conservación, restauración o sustitución", y "prevenir y controlar los factores de deterioro ambiental, imponer las sanciones legales y exigir la reparación de los daños causados" (Constitución Política, 1991, art. 80).

En este sentido, se evidencia que la Carta Política de 1991 reconoció que el derecho fundamental al medio ambiente sano tiene el carácter de interés superior, el cual se ha desarrollado en alrededor de treinta (30) disposiciones "que consagran una serie de principios, mandatos y obligaciones enfocados en una doble dimensión dirigida a: (i) proteger de forma integral el medio ambiente y (ii) garantizar un modelo de desarrollo sostenible, sobre los que se ha edificado el concepto de "Constitución Ecológica"' (Corte Constitucional, 2016, Sentencia T-622). Por ello, según el Alto Tribunal Constitucional, el medio ambiente desde su dimensión objetiva, corresponde a un derecho colectivo, pero a su vez, es de carácter subjetivo y fundamental, "al resultar ligado indefectiblemente con los derechos individuales a la vida y a la salud de las personas" (Corte Constitucional, 2017, Sentencia T-325). Derivado de lo anterior, le son impuestas obligaciones a los poderes públicos para que este derecho pueda ser respetado, protegido y garantizado.

No obstante, el reconocimiento del derecho al medio ambiente ha generado como mínimo dos problemas esenciales desde al ámbito constitucional: por una parte, la existencia de interrelaciones recíprocas entre el derecho al medio ambiente y ciertos derechos de rango constitucional, especialmente el derecho de propiedad; por otra parte, la configuración jurídica de estas interrelaciones por parte del legislador y, eventualmente, por los jueces ante situaciones concretas (Gomes, 1995). Para ello, en términos generales, se ha planteado aplicar el EBDH, en virtud del cual

se identifica a los titulares de derechos y aquello a lo que tienen derecho, así como las correspondientes instancias de protección o titulares de deberes y las obligaciones que les incumben, y se procura fortalecer la capacidad de que gozan los primeros. (ACNUDH \& Cepal, 2016, p. 19)

De ahí que las acciones estatales deben dirigirse a la materialización de los derechos, en el que "los estándares internacionales de derechos humanos deben constituir guías y pautas aplicables a las temáticas o problemas del desarrollo" (ACNUDH \& Cepal, 2016, p. 19). 
En otras palabras, el derecho al medio ambiente solo puede garantizarse si el ordenamiento jurídico es capaz de relacionarlo con otros derechos, siguiendo los lineamientos internacionales en derechos humanos, con una perspectiva de desarrollo sostenible, garantizando la seguridad humana. En este sentido, la Agenda 2030 para el desarrollo sostenible está dirigida a fortalecer la interconexión entre derechos humanos y desarrollo sostenible en términos de universalidad, participación e inclusión, teniendo en cuenta que la igualdad es el eje articulador del desarrollo, con miras a erradicar la pobreza extrema, las desigualdades y todas las amenazas que hoy el individuo enfrenta en cuanto riesgos a su seguridad humana.

Por lo anterior, es importante verificar si se está construyendo un sistema que garantice la justicia ambiental (Bellver, 1996) con base en tres principios planteados por Saladin (1995) y Häberle (1998): en primer lugar, la justicia intrageneracional frente a los individuos que comparten el mismo periodo de vida; en segundo lugar, la justicia intergeneracional frente a las generaciones futuras, y finalmente la justicia frente al medio ambiente natural. Por tanto, se pasa a analizar el derecho al medio ambiente desde la óptica de la seguridad humana.

\section{Derecho al medio ambiente en el marco de la seguridad humana}

Los pilares fundamentales de la ONU (2012) —desarrollo humano, derechos humanos y paz y seguridad - deben interrelacionarse y reforzarse entre sí con miras a garantizar los fines de la comunidad internacional y de cada uno de los Estados miembro. La Declaración Universal de los Derechos Humanos proclama que uno de los propósitos de la ONU es "desarrollar y estimular el respeto de los derechos humanos y las libertades fundamentales de todas las personas", y reconoce su interdependencia con la paz, la seguridad y el desarrollo" (ACNUDH \& Cepal, 2016, p. 17).

Así, es evidente que desde el nacimiento mismo de la ONU se tenía claro que, para mantener el mundo en paz, era necesario garantizar la seguridad, en términos generales, a los Estados, pero concretamente a los individuos frente a las amenazas que enfrentaran en torno a sus derechos. A pesar de ello, no ha existido una verdadera interrelación y articulación de los pilares que pretendían dirigir las acciones de la comunidad internacional, por lo cual se ha desarrollado cada uno de ellos por separado. Con ello, tanto el desarrollo económico como la seguridad y los derechos humanos tenían unas dinámicas no solo disímiles, sino en muchos casos contradictorias.

Esto se hizo evidente, por ejemplo, en el desarrollo basado en el crecimiento económico infinito con una producción creciente, a costa de una afectación al entorno, modelo que apenas se empezó a replantear desde el informe Brundtland (1987), pero que solo se ha concretado profundamente con los ODS de 2015, aunque con un grado de vinculatoriedad muy bajo, a pesar de que el derecho a un ambiente sano se proclamó como derecho humano a partir de 1972. 
Esta misma situación se presentó respecto a la seguridad con miras al mantenimiento de la paz, que se entendía en la comunidad internacional con una dinámica propia. Después de la Segunda Guerra Mundial, la seguridad se desarrolló bajo el denominado paradigma realista, según el cual se encuentra vinculada al poder estatal (Estella et al., 2010) bajo el concepto de seguridad nacional.

La perspectiva de la seguridad nacional, planteada por Kennan (1948) y adoptada por casi todos los Estados, se refería a "la capacidad continuada de un país para proseguir el desarrollo de su vida interna sin interferencia seria, o amenaza de interferencia de potencias extranjeras". Por ello, la seguridad dependía casi que exclusivamente del poder militar, con miras a garantizar la subsistencia de los Estados, o para contrarrestar o disuadir las amenazas por parte de otros (Laborie, 2011). Así, la perspectiva realista de seguridad nacional tenía como objetivo prevenir o rechazar las amenazas de naturaleza militar, razón por la que se orientaba a proteger militarmente la soberanía, la independencia y el espacio físico del Estado frente a los agresores potenciales (Font \& Ortega, 2012).

En consecuencia, la seguridad de los individuos solo podía garantizarse si se podía garantizar la seguridad del Estado desde la perspectiva militar, que tenía como objeto de protección principal al territorio, frente a la amenaza de una agresión militar extranjera, concretamente por parte de otro u otros Estados (Estella et al., 2010). Esta visión fue claramente manifiesta durante el periodo de la Guerra Fría, en el que se enfrentaban dos grandes bloques de países liderados por las superpotencias mundiales de Estados Unidos y la Unión Soviética. Para Leal (2003), el enfoque de seguridad nacional "ha estado determinado por este origen y fue influenciado por la estrategia estadounidense de contención. La ideología del anticomunismo, propia de la Guerra Fría, le dio sentido, y la desconfianza entre las naciones le proporcionó su dinámica” (p. 74).

Sin embargo, con la finalización de la Guerra Fría, se empezó a construir un nuevo orden mundial en el que los escenarios y la naturaleza de los conflictos bélicos mutaron significativamente. Primero, ya no se enmarcaban en un enfrentamiento ideológico polarizado entre los bloques liderados por las potencias. Segundo, y como consecuencia de lo primero, tanto la financiación como el apoyo militar que recibían quienes eran parte de un conflicto alrededor del mundo disminuyeron significativamente y en algunos casos se cancelaron por completo. Tercero, como resultado, la naturaleza de los conflictos también mutó a conflictos internos o, en el peor de los casos, a guerras civiles. Por último, estos conflictos se agudizaron por la ausencia de lineamientos ideológicos y la necesidad de financiamiento para mantener o alcanzar el poder militar a través de actividades que generaban afectaciones significativas a la población civil y al medio ambiente (Font \& Ortega, 2012). Como consecuencia de estos conflictos armados internos, las violaciones a los derechos de los individuos se incrementaron, la pobreza se agudizó y la gobernabilidad de los Estados se tornó cada vez más compleja, lo que hacía necesario replantearse las políticas en materia de desarrollo, seguridad y derechos humanos. 
Para responder a esta necesidad de cambio, surgieron dos perspectivas bien diferenciadas. Una primera corriente que mantiene la visión estado-céntrica como eje de la seguridad, con base en lo cual propone la inclusión de una serie de factores que complementan el concepto de seguridad nacional. Entre estas propuestas se destacan los conceptos de seguridad común (Palme, 1982), seguridad con fines defensivos (ONU, 1993) y los de seguridad integral de la ASEAN y seguridad cooperativa (Laborie, 2011).

La segunda corriente propone un cambio de visión en torno al objeto de la seguridad centrándola en el individuo (Laborie, 2011). Con ello se dirige hacia una perspectiva idealista de la seguridad, vinculada directamente con el concepto de paz (Estella et al., 2010). Esta corriente propone abandonar la hegemónica visión estado-céntrica de la seguridad al orientarla hacia el individuo como auténtico sujeto de derecho internacional. De esta forma se puede materializar lo planteado por Locke (2006) respecto a la seguridad como razón de ser de la organización estatal, con lo cual "la seguridad se convierte en el primer derecho del individuo y, en consecuencia, en la primera y más básica obligación del Estado respecto de sus ciudadanos" (Ortega, 2010, p. 5). Desde esta perspectiva, aunque el Estado se considera uno de los principales actores de la política internacional, no es el único; en las relaciones internacionales confluyen un sinnúmero de actores subnacionales $y$ transnacionales que ejercen influencia en mayor o menor medida sobre dichas relaciones (Orozco, 2006), por lo cual deben enfocarse necesariamente en los individuos.

A partir de esa propuesta, la seguridad debería estar dirigida a la protección de los individuos más que a la protección del territorio, pasando de la seguridad a través de las armas a la seguridad por medio del desarrollo humano sostenible. Con esta lógica, el PNUD, en el Informe sobre el Desarrollo Humano de los ańos 1993 y 1994, adoptó el concepto de seguridad humana, entendida por la Comisión de Seguridad Humana (2003) de la siguiente manera:

Consiste en proteger la esencia vital de todas las vidas humanas de una forma que realce las libertades humanas y la plena realización del ser humano. Seguridad humana significa proteger las libertades fundamentales: libertades que constituyen la esencia de la vida. Significa proteger al ser humano contra las situaciones y las amenazas críticas (graves) y omnipresentes (generalizadas). Significa la creación de sistemas políticos, sociales, medioambientales, económicos, militares y culturales que, en su conjunto, brinden al ser humano las piedras angulares de la supervivencia, los medios de vida y la dignidad. (p. 3)

Así las cosas, para un individuo, la seguridad se relaciona con "la protección contra la amenaza de la enfermedad, el hambre, el desempleo, el delito, el conflicto social, la represión política y los riesgos del medio ambiente" (PNUD, 1994, p. 25). La seguridad humana se refiere, entonces, a la libertad para vivir sin miedo, sin miseria y en dignidad (PNUD, 2011). Por tal razón, este nuevo concepto permitió replantear los discursos paralelos en torno a la seguridad y el desarrollo, ya que, con el abandono de la visión estado-céntrica y la adopción de una visión centrada en el individuo, "se resaltaba la tensión 
existente entre promover la seguridad de Estado y promover la seguridad de los individuos, que históricamente ha sido puesta en peligro por el Estado" (Krause, 2005, pp. 21-22).

La seguridad humana tiene un carácter multidimensional y ha sido presentada como un "concepto polivalente: de contenido antropocéntrico, universal, interdependiente, preventivo, democrático, indivisible, global, local, integrativo y de connotaciones cualitativas y cuantitativas" (Villanueva, 2000, p. 106). En este sentido, el PNUD (1994) ha establecido que se compone de una serie de elementos o dimensiones que se interrelacionan entre sí: la seguridad económica, la seguridad alimentaria, la seguridad en materia de salud, la seguridad ambiental, la seguridad personal, la seguridad de la comunidad y la seguridad política. Cada uno de estos elementos o dimensiones responde a una serie de amenazas como la pobreza, el hambre, la violencia o la contaminación, entre otros (Mack, 2005). A partir de estos elementos, es evidente que la seguridad humana se centra en la protección de los individuos, de las comunidades y del ambiente. Por su carácter integrado y su multidimensionalidad, responde a temáticas que no se habían abordado tradicionalmente en los niveles de seguridad nacional y seguridad internacional (Rojas, 2007). Así, mientras que la seguridad nacional responde a la perspectiva tradicional de proteger a los Estados frente a amenazas o agresiones externas, la seguridad humana se dirige a la protección del individuo, sin importar su nacionalidad, en sus diferentes dimensiones (Estella et al., 2010).

En cuanto a la seguridad ambiental, ha tenido un desarrollo teórico importante como uno de los elementos esenciales de la seguridad humana, a partir de lo planteado por Ullman (1983). Este autor intentó redefinir el concepto de seguridad ampliando las amenazas, directas o indirectas, a la seguridad de los Estados y los individuos, al incluir variables distintas a las militares, como los desastres naturales o los problemas de abastecimiento de recursos naturales, entendidas como amenazas internas a los Estados (Lavaux, 2004). A partir de este planteamiento de Ullman (1983), desarrollado por Buzan (1991) y Buzan y Weaver (1997), se ha logrado identificar, desde la perspectiva multidimensional, por lo menos tres generaciones de estudios enfocados en la seguridad ambiental derivados de la identificación y sistematización de una serie de riesgos para el medio ambiente.

Una primera generación de estudios que relacionaron la seguridad con el aspecto medioambiental se remite a la década de los ochenta. Estos planteaban la necesidad de incluir el factor ambiental en una versión más amplia del concepto de seguridad nacional, sin referirse concretamente al concepto de seguridad ambiental. Sin embargo, estos estudios no fueron lo suficientemente analíticos, y al encontrarse sin comprobación empírica fueron seriamente cuestionados (Lavaux, 2004).

En este escenario, en la década de los noventa surgió la segunda generación de estudios de la seguridad ambiental, liderados por el denominado Grupo de Toronto, con la intención de demostrar empíricamente la relación entre seguridad y medio ambiente o, como lo plantearon, entre "escasez de recursos y conflictos inter e intraestatales" (Lavaux, 2004, p. 95). Dentro de estos estudios se destacan los de Homer-Dixon (1994), quien 
manifestaba, por ejemplo, que las generaciones futuras sufrirían el agotamiento y degradación de acuíferos, ríos y otras fuentes de agua, y quizás un cambio climático significativo; si la escasez de estos recursos fuera severa, podría precipitar violencia civil e incluso conflictos internacionales. En este sentido, sus estudios se centraron en identificar las complejas relaciones causales entre la escasez ambiental, el conflicto interno y los efectos sociales como pobreza y migraciones.

Esta segunda ola de estudios sobre seguridad ambiental determinó que el deterioro de la calidad del medio ambiente, entendido como escasez de recursos renovables, podía empeorar otros factores políticos, sociales o económicos que a su vez podían ser la causa de conflictos violentos (Unión Internacional por la Conservación de la Naturaleza [IUCN], 1999, p. 26). Sin embargo, el determinismo de las investigaciones adelantadas en esta segunda generación fue bastante limitado, ya que en la mayoría de casos no era posible atribuirle la causa única de los conflictos políticos o incluso de los conflictos medioambientales a los cambios en el medio ambiente. Por ello, esta segunda ola se centró en estudiar los conflictos ambientalmente inducidos (IUCN, 1999).

Finalmente, una tercera generación, crítica de la anterior, manifiesta que, durante la segunda generación, los investigadores se preguntaron solamente si el medio ambiente podría contribuir al conflicto, pero no se ocuparon de entender por qué en algunos casos sí lo hacía y en otros no (Dabelko, 1999). Por ello, se planteó el retorno a una amplia gama de variables dependientes, que relacionan la seguridad ambiental con las demás dimensiones de la seguridad humana (Levy, 1994; Barnett, 2000).

Esto demuestra indefectiblemente que el proceso de determinación del concepto mismo y el campo de acción de la seguridad ambiental no ha sido unánime ni ha estado libre de críticas. Al parecer, hoy en día se perfila una cuarta generación de estudios que intenta sintetizar los planteamientos de las generaciones anteriores, al entender "las relaciones entre el medio ambiente y la seguridad en términos multidimensionales tanto de conflicto como de cooperación y de seguridad humana, tanto para los Estados, así como para las sociedades y las personas" (Lavaux, 2004, p. 97).

Para finalizar, es importante, entonces, tener en cuenta que, en ese proceso de evolución y cambio de paradigma de la seguridad hasta el concepto de seguridad humana hoy en día, se ha incorporado la seguridad ambiental como una de sus dimensiones. La seguridad ambiental, igualmente, ha ido evolucionando desde la década de los ochenta y no cabe duda, pese a la discusión que ha generado, de que tiene una alta relevancia desde esta "nueva" perspectiva de la seguridad.

\section{Principios marco sobre los derechos humanos y el medio ambiente}

Como se evidencia, el reconocimiento del medio ambiente sano como derecho humano ha sido relativamente reciente, y su proceso de internacionalización se concreta con "la 
adopción de varios instrumentos declarativos y convencionales en el seno de las Naciones Unidas, y la creación progresiva de mecanismos regionales e internacionales protectores de los derechos humanos" (Bruzón \& Antúnez, 2012, p. 110).

Sin embargo, la incorporación de este derecho no ha sido uniforme en los Estados. Loperena (1998) manifiesta, por ejemplo, que la internacionalización necesaria de la protección del medio ambiente, que podría acortar dichas diferencias y generar una armonización de las legislaciones nacionales, no ha sido ni suficiente ni idónea. En el plano jurídico, es común apreciar que "las constituciones recogen principios ambientales, mandatos a los poderes públicos e incluso proclaman derechos. El cumplimiento cabal de los presupuestos ambientalistas insertos en las constituciones reclama esa transformación profunda y radical de un sistema económico globalizado" (Canosa, 2006, p. 159).

La relación del derecho al medio ambiente sano con otros derechos como la vida digna, la salud, el derecho a la tierra (Torres \& Aguilar, 2018) o el trabajo ha sido objeto de desarrollo en las legislaciones y en la jurisprudencia de las altas cortes tanto nacionales como internacionales, teniendo en cuenta la interdependencia entre estos derechos. A nivel interno, incluso se ha llegado a reconocer elementos de la naturaleza como sujetos de derechos, como en el caso del río Atrato (Corte Constitucional, 2016, Sentencia T-622), la Amazonía (Corte Suprema de Justicia, 2018, Sentencia 4360) y el parque Isla de Salamanca (Corte Suprema de Justicia, 2020, Sentencia 3872). Por su parte, desde la perspectiva internacional, Calderón (2017) se ha referido al papel de los tribunales internacionales en los siguientes términos:

Los mecanismos jurídicos internacionales, aunque demorados, están representando una ventana de oportunidad para documentar y presentar casos que evidencian afectaciones particulares en materia ambiental. El Sistema Interamericano de Derechos Humanos (SIDH) se presenta ahora como una alternativa regional para el análisis de casos o situaciones particularmente importantes en esta área para la región americana. (p. 103)

Con este panorama, el primer relator especial de las Naciones Unidas sobre los Derechos Humanos, John Knox (ACNUDH, 2018), presentó al Consejo de los Derechos Humanos de las Naciones Unidas los denominados Principios Marco sobre los Derechos Humanos y el Medio Ambiente. Estos dieciséis principios marco (PM) parten de la relación simbiótica entre los derechos humanos y el medio ambiente, ya que, por una parte, los Estados deben garantizar un medio ambiente sin riesgos, limpio, saludable y sostenible con el fin de respetar, proteger y hacer efectivos los derechos humanos (PM 1), pero a su vez deben respetar, proteger y hacer efectivos los derechos humanos con el fin de garantizar un medio ambiente sin riesgos, limpio, saludable y sostenible (PM 2).

Con estos postulados, se hace evidente la relación de estos principios con la perspectiva de la seguridad humana, pues, como se ha descrito, esta visión de seguridad implica la protección al ser humano frente a las situaciones y las amenazas críticas y generalizadas. 
Así, la seguridad humana no es otra cosa que la creación de una serie de sistemas de diversa índole (político, social, medioambiental, económico, militar y cultural) que garanticen al ser humano la supervivencia, los medios de vida y la dignidad (Comisión de Seguridad Humana, 2003).

De esta forma, el primer principio se relaciona directamente con la seguridad ambiental para el disfrute de los derechos humanos, y el segundo principio con las demás dimensiones de la seguridad humana, para el disfrute del medio ambiente. Con ello, la ONU (2018) finalmente estructuró unos lineamientos para que los Estados logren armonizar los conceptos de seguridad humana y derechos humanos frente un factor tan trascendental como el medio ambiente.

En general, todos los principios se encuentran relacionados de manera directa o indirecta con los derechos humanos, el medio ambiente y las dimensiones de la seguridad humana. Los principios recogen obligaciones diversas, como la prohibición de la discriminación y la obligación de garantizar una protección igual y efectiva en relación con el disfrute de un medio ambiente (PM 3), en un entorno seguro para las personas, los grupos y los órganos de la sociedad que se ocupan de los derechos humanos y/o los asuntos medioambientales (PM 4). Asimismo, está la exigencia de respetar y proteger la libertad de expresión, asociación y reunión pacífica (PM 5), proporcionando acceso al público a la información ambiental de manera asequible, efectiva y oportuna (PM 7), y permitiendo su participación en el proceso de toma de decisiones en torno a aspectos relacionados con el medio ambiente (PM 9).

Así mismo, los Estados deben estipular normas ambientales sustantivas que no sean discriminatorias y no tengan carácter regresivo, diseñadas para proteger los derechos humanos (PM 11) y garantizar su aplicación efectiva por parte de las entidades públicas y el sector privado (PM 12). Para ello, se exige, entre otras cosas, la evaluación previa de los eventuales impactos ambientales de las políticas y proyectos, con miras a proteger los derechos de los más vulnerables al daño ambiental o a quienes se encuentren en una situación de especial riesgo (PM 14), como los pueblos indígenas y los miembros de las comunidades tradicionales (PM 15).

Así, es claro que, en mayor o menor medida, todos estos principios se refieren a los diferentes tipos de riesgos que la seguridad humana pretende proteger desde una perspectiva multidimensional. En este sentido, la seguridad ambiental se relaciona de forma evidente con todos los principios de manera directa, pero también hay relaciones entre algunos de los principios y la seguridad económica, alimentaria y de salud (PM 12 y 16), la seguridad personal (PM 4), la seguridad de la comunidad (PM 14 y 15) y la seguridad política (PM 7 y 9).

Finalmente, el último de los principios marco señala que los Estados deben respetar, proteger y hacer efectivos los derechos humanos en el marco de las medidas que adopten para hacer frente a los problemas ambientales y alcanzar el desarrollo sostenible (PM 16), lo cual también implica una armonización con el "nuevo" paradigma de desarrollo. 
En últimas, estos principios marco evidencian la coordinación y la armonización de los tres pilares de acción de la ONU, que se concretan en el ámbito medioambiental. En cuanto al primer pilar, el desarrollo, este se ha orientado hacia el desarrollo sostenible, sustentado en tres dimensiones: económica, ambiental y social. En cuanto al segundo pilar, los derechos humanos, no solo se ha reconocido el derecho al medio ambiente como derecho humano, sino que se ha establecido, especialmente con estos principios marco, una relación simbiótica con los derechos humanos en general. Finalmente, en cuanto al tercer pilar, la paz y la seguridad, el cambio del paradigma de la seguridad hacia la seguridad humana ha reconocido como esencial la seguridad ambiental.

Desde esta perspectiva, los principios marco marcan un punto de partida para los Estados en el camino de armonizar todas sus obligaciones enmarcadas en los conceptos de desarrollo sostenible, derechos humanos y seguridad humana, en aras de materializar una verdadera justicia ambiental.

\section{Conclusiones}

El medio ambiente ha sido una preocupación de las sociedades que ha impregnado los ordenamientos jurídicos desde el Imperio romano, pero solo hasta hace algunas décadas ha sido considerado un bien jurídico, objeto de protección en el derecho internacional de los derechos humanos y, consecuentemente, en los diferentes ordenamientos internos de los Estados.

Así mismo, se ha incorporado como una de las dimensiones en las dinámicas del desarrollo sostenible y la seguridad humana, con lo cual constituye un elemento transversal en los tres pilares de acción de la más importante organización de la comunidad internacional, la ONU. Esta incorporación, además de lenta y paulatina, ha sido bastante controversial en todos los aspectos (derechos humanos, desarrollo y seguridad), aunque en algunos más que en otros. Sin embargo, no cabe duda de que hoy los asuntos ambientales son considerados esenciales en cada uno de estos aspectos.

Concretamente, desde la perspectiva de la seguridad humana, la dimensión de la seguridad ambiental ha tomado una relevancia especial, por cuanto es necesaria para garantizar el desarrollo sostenible, en virtud de que uno de sus aspectos es el ecológico, así como para garantizar los derechos humanos, tal como lo evidencian los principios marco estudiados. Estos principios, que agrupan las principales obligaciones de los Estados en materia de derechos humanos y medio ambiente, se relacionan clara y directamente con la seguridad ambiental, así como con cada una de las demás dimensiones de la seguridad humana, dada su perspectiva multidimensional.

De esta manera, los principios marco sobre los derechos humanos y el medio ambiente constituyen un elemento clave para armonizar todas las obligaciones estatales de cara al desarrollo sostenible, los derechos humanos y la seguridad humana. 


\section{Declaración de divulgación}

Los autores declaran que no existe ningún potencial conflicto de interés relacionado con el artículo. El artículo es un producto de investigación del Grupo Memoria Histórica, Construcción de Paz, Derechos Humanos, DICA y Justicia de la Escuela Superior de Guerra, desarrollado dentro del proyecto de investigación "Esclarecimiento de la verdad histórica sobre la violencia estructural en Colombia, provocada al medio ambiente y a las víctimas del conflicto”.

\section{Financiamiento}

Los autores declaran como fuente de financiamiento para la realización de este artículo la Escuela Superior de Guerra.

\section{Sobre los autores}

Mauricio Antonio Torres Guarnizo es Legum Magister de la Universidad de Konstanz (Alemania), administrador de empresas de la Universidad Militar Nueva Granada y abogado de la Universidad Santo Tomás. Investigador del Grupo Memoria Histórica, Construcción de Paz, Derechos Humanos, DICA y Justicia de la Escuela Superior de Guerra (Colombia).

https://orcid.org/0000-0003-0487-7283 - Contacto: mauricio.torres@esdegue.edu.co

Elmers Freddy Velandia Pardo es candidato a doctor en Derecho, Educación y Desarrollo, máster en derechos humanos, máster MBA, internacionalista y politólogo. Es investigador junior (Minciencias). Es docente e investigador de la Escuela Superior de Guerra (Colombia).

https://orcid.org/0000-0003-1217-9178 - Contacto: elmers.velandia@esdegue.edu.co

\section{Referencias}

Acero, J. (2009, 14 de junio). Destacan el espíritu ecológico de los Romanos. La Tercera. https://bit.ly/3CAfotd Alkire, S. (2003). A conceptual framework for human security (CRISE Working Paper 2, Centre for Research on Inequality, Human Security and Ethnicity, Queen Elizabeth House, University of Oxford). https:// bit.ly/3Cwd2vq

Barnett, J. (2000). Destabilizing the environment conflict thesis. Review of International Studies, 26(2), 271-288. https://www.jstor.org/stable/20097674

Bellver, V. (1996). El movimiento por la justicia ambiental: entre el ecologismo y los derechos humanos. En Anuario de Filosofía del Derecho XIII (pp. 327-347). Universidad de Valencia.

Bruzón, C., \& Antúnez, A. (2012). Reflexiones en torno a la protección de los derechos humanos desde la perspectiva del derecho ambiental internacional. Producción + Limpia, 7(2), 106-117. https://bit. ly/3x5JC6e

Buzan, B. (1991). People, states and fear. An agenda for international security studies in the post-Cold War era. Review of International Studies, 23, 313-326.

Buzan, B., \& Weaver, O. (1997). Slippery? Contradictory? Sociologically untenable? The Copenhagen school replies. International Affairs, 67(3), 431-451. https://doi.org/10.1017/S0260210597002416 
Canosa, R. (2006). ¿Existe un verdadero derecho constitucional a disfrutar del medio ambiente? Anuario de Derechos Humanos, 7(1), 151-215.

Comisión Brundtland. (1987). Nuestro futuro común (informe).

Comisión de Seguridad Humana. (2003). La seguridad humana ahora. CHS.

Constitución Política de Colombia. (1991). Gaceta Constitucional n. ${ }^{\circ}$ 116. http://bit.ly/2NA2BRg

Corte Constitucional de Colombia. (1992, 5 de junio). Sentencia T-406 (Ciro Angarita, M. P.). https://bit. ly/3x8abrA

Corte Constitucional de Colombia. (1992, 17 de junio). Sentencia T-415 (Ciro Angarita, M. P.). https://bit. ly/3DEq1MU

Corte Constitucional de Colombia. (1998, 2 de diciembre). Sentencia SU-747 (Eduardo Cifuentes, M. P.). https://bit.ly/3nDmed4

Corte Constitucional de Colombia. (2016, 10 de noviembre). Sentencia T-622. (Jorge Palacio, M. P.). https:// bit.ly/3nRu3fk

Corte Suprema de Justicia. (2018, 5 de abril). Sentencia 4360. (Luis Tolosa, M. P.). https://bit.ly/3DUH2Ti

Corte Suprema de Justicia. (2020, 18 de junio). Sentencia 3872. (Octavio Tejeiro, M. P.). https://bit.ly/3CTNngc

Dabelko, D. (1999). The environmental factor. The Wilson Quartely, 23(4), 14-19. https://bit.ly/3cyrB7d

De Castro, B. (2003). La clasificación de los derechos humanos. En B. de Castro (Coord.), Introducción al estudio de los derechos humanos. Editorial Universitas.

Decreto 2811. (1974, 18 de diciembre). Por el cual se dicta el Código Nacional de Recursos Naturales Renovables y de Protección al Medio Ambiente. Presidencia de la República de Colombia. https:// bit.ly/3cC09pe

Delgado, F. (1993). Régimen del derecho constitucional al medio ambiente. Revista Española de Derecho Constitucional, 13(38), 49-80.

Estella, A., Torres, A., \& Cebada, A. (2010). El debate sobre la estrategia de seguridad española. Fundación Ideas. https://dialnet.unirioja.es/servlet/libro?codigo $=572577$

Font, T., \& Ortega, P. (2012). Seguridad nacional, seguridad multidimensional, seguridad humana. Papeles de Relaciones Ecosociales y Cambio Global, 119, 161-172. https://bit.ly/2Z88Zb7

Gomes Canotilho, J. (1995). Protecção do ambiente e direito de propriedade. Coimbra Editora.

Häberle, P. (1998). Ein Verfassungsrecht für künftige Generationen. Die “andere” Form des Gesellschaftsvertrages: der Generationenvertrag. En Verfassung, Theorie und Praxis des Sozialstaats. Festschrift für Hans F. Zacher zum 70 (pp. 215-233). Geburtstag.

Homer-Dixon, T. (1994). Environmental scarcities and violent conflict, evidence from cases. International Security, 19(1), 6-7.

Krämer, L. (1999). Derecho ambiental y Tratado de la Comunidad Europea. Marcial Pons.

Krause, K. (2005). Seguridad humana: ¿ha alcanzado su momento? Revista Papeles de Cuestiones Internacionales, 90, 19-30. https://bit.ly/3FAXo3W

Laborie, M. (2011). Documento Marco del Instituto Español de Estudios Estratégicos. IEES.

Landa, C. (2017). Principios de la Constitución Ambiental. En A. Cançado \& C. Barros (Coords.), Derechos humanos y medio ambiente (pp. 27-47). Expressão Gráfica e Editora. https://bit.ly/3r0vn1x

Lavaux, S. (2004). Medio ambiente y seguridad: una relación controvertida pero necesaria. En D. Cardona, B. Labatut, S. Lavaux, \& R. Sánchez (Eds.), Encrucijada de la seguridad en Europa y las Américas. Centro de Estudios Políticos Internacionales, Universidad del Rosario. 
Leal, F. (2003). La doctrina de seguridad nacional: materialización de la Guerra Fría en América del Sur. Revista de Estudios Sociales, 15, 74-87. https://doi.org/10.7440/res15.2003.05

Levy, M. (1994). Time for a third wave of environment and security scholarship? Environmental Change and Security Project Report, 1, 44-46. https://bit.ly/3x7Lmfo

Locke, J. (2006). Segundo tratado sobre el Gobierno civil. Tecnos.

Loperena, D. (1998). Los principios del derecho ambiental. Civitas.

Mack, A. (2005). El concepto de seguridad humana. Papeles de Cuestiones Internacionales, 90, 11-18. https://bit.ly/3cBAgWA

Morrillas, P. (2006). Génesis y evolución de la expresión de la seguridad humana. Revista CIDOB d'Afers Internacionals, 76, 47-58. https://bit.ly/3cxWE2W

Muńoz, E. (2017). El medio ambiente como bien jurídico y derecho humano de tercera generación reconocido desde el Imperio romano. En A. Cançado \& C. Barros (Coords.), Derechos humanos y medio ambiente (pp. 83-102). Expressão Gráfica e Editora. https://bit.ly/3r0vn1x

Oficina del Alto Comisionado para los Derechos Humanos (ACNUDH). (2018). Principios Marco sobre los Derechos Humanos y el Medio Ambiente. https://bit.ly/3DB1NTM

Oficina del Alto Comisionado para los Derechos Humanos (ACNUDH) \& Comisión Económica para América Latina y el Caribe (Cepal). (2016). Sociedad, derechos y medio ambiente. Estándares internacionales de derechos humanos aplicables al acceso a la información, a la participación pública y al acceso a la justicia. https://bit.ly/3qh5WGT

Organización de las Naciones Unidas (ONU). (1948). Declaración Universal de Derechos Humanos. https:// bit.ly/3CIgxyS

Organización de las Naciones Unidas (ONU). (1966). Pacto Internacional de Derechos Económicos, Sociales y Culturales. https://bit.ly/30LkiGy

Organización de las Naciones Unidas (ONU). (1972). Declaración de las Naciones Unidas sobre el Medio Humano. En Informe de la Conferencia de las Naciones Unidas sobre el Medio Humano (Estocolmo). https://bit.ly/3oNyGq3

Organización de las Naciones Unidas (ONU). (2012, 10 de septiembre). Resolución 66/290. Seguimiento del párrafo 143, relativo a la seguridad humana, del Documento Final de la Cumbre Mundial 2005. https://digitallibrary.un.org/record/737105? $\mathrm{ln}=\mathrm{en}$

Orozco, G. (2006). El concepto de seguridad en la teoría de las relaciones internacionales. Revista CIDOB d'Afers Internacionals, 76. https://bit.ly/30F67mp

Ortega, M. (2010). Introducción. En De las operaciones conjuntas a las operaciones integradas. Un nuevo desafio para las Fuerzas Armadas (pp. 7-11). Centro Superior de Estudios de la Defensa Nacional; Ministerio de Defensa de España.

Pérez, E. (2007). Derechos humanos y medio ambiente. Bioètica \& Debat, 48, 7-10. https://bit.ly/3nDtS7x Programa de las Naciones Unidas para el Desarrollo (PNUD). (1994). Informe sobre Desarrollo Humano. http://hdr.undp.org/en/content/human-development-report-1994

Programa de las Naciones Unidas para el Desarrollo (PNUD). (2011). El enfoque de la seguridad humana desde tres estudios de caso. Instituto Interamericano de Derechos Humanos.

Programa de las Naciones Unidas para el Desarrollo (PNUD). (2015). Objetivos de Desarrollo Sostenible (ODS). https://bit.ly/3x9uGUE

Rodríguez, J. (2017). Los derechos humanos y el medio ambiente. En A. Cançado \& C. Barros (Coords.), Derechos humanos y medio ambiente (pp. 173-190). Expressão Gráfica e Editora. https://bit. ly/3r0vn1x 
Rodríguez Palop, M. (2002). La nueva generación de derechos humanos. Origen y justificación. Editorial Dykinson.

Rojas, F. (2007). Seguridad humana: aportes en la reformulación del concepto de seguridad. Flacso.

Saladin, P. (1995). Wozu noch Saaten? Verlag Stämpflicie AG.

Torres, M. \& Aguilar, A. (2018). El derecho a la tierra en Colombia. En M. Torres (Ed.), Diálogos: los derechos humanos después del Acuerdo (pp. 127-155). Escuela Superior de Guerra "General Rafael Reyes Prieto". https://bit.ly/3CFtXvI

Ullman, R. (1983). Redefining security. International Security, 8(1).

Unión Internacional por la Conservación de la Naturaleza (IUCN). (1999). State-of-the-Art review on environment, security and development co-operation. https://bit.ly/3yJIRAw

Vasak, K. (Ed.). (1984). Las dimensiones internacionales de los derechos humanos. Serbal; Unesco.

Villanueva, M. (2000). La seguridad humana: ¿una ampliación del concepto de seguridad global? Revista Mexicana de Politica Exterior, 59, 106-130. https://bit.ly/3yMQiaj 